\title{
Delocalized and localized charged excitons in single CdSe/CdS dot-in- rods revealed by polarized photoluminescence blinking
}

\section{$\operatorname{AUTHOR}(\mathrm{S}):$}

Ihara, Toshiyuki; Sato, Ryota; Teranishi, Toshiharu; Kanemitsu, Yoshihiko

\section{CITATION:}

Ihara, Toshiyuki ...[et al]. Delocalized and localized charged excitons in single CdSe/CdS dot-in-rods revealed by polarized photoluminescence blinking. Physical Review B 2014, 90(3): 035309.

\section{ISSUE DATE:}

\section{4-07-15}

\section{URL:}

http://hdl.handle.net/2433/189408

RIGHT:

C2014 American Physical Society 
PHYSICAL REVIEW B 90, 035309 (2014)

\title{
Delocalized and localized charged excitons in single $\mathrm{CdSe} / \mathrm{CdS}$ dot-in-rods revealed by polarized photoluminescence blinking
}

\author{
Toshiyuki Ihara, Ryota Sato, Toshiharu Teranishi, and Yoshihiko Kanemitsu* \\ Institute for Chemical Research, Kyoto University, Uji, Kyoto 611-0011, Japan \\ (Received 23 December 2013; revised manuscript received 24 June 2014; published 15 July 2014)
}

\begin{abstract}
$\mathrm{CdSe} / \mathrm{CdS}$ heterostructured nanocrystals with quasi-type-II band alignments provide an interesting platform for studying the photoluminescence (PL) blinking associated with their unique morphologies. By using simultaneous measurements of the PL intensity, lifetime, and polarization anisotropy, we reveal the role of the electron delocalization during the blinking of single $\mathrm{CdSe} / \mathrm{CdS}$ dot-in-rods. We found that a significant change in the PL polarization anisotropy distinguishes between two kinds of charged excitons with different electron delocalizations. We report our observation of unique polarized PL blinking governed by the band alignments and the Coulomb interactions between the charges inside and outside the dot-in-rod.
\end{abstract}

DOI: 10.1103/PhysRevB.90.035309

\section{INTRODUCTION}

Photoluminescence (PL) intermittency, also known as blinking, has been the subject of intensive research since its first observation in single semiconductor nanocrystals (NCs) in 1996 [1]. A good understanding of the blinking mechanism is important for future applications of stable single-photon sources for quantum communication and cryptography [2]. In addition to the widely studied $\mathrm{CdSe} / \mathrm{ZnS}$ core-shell NCs, recent advances in synthesis techniques have realized the fabrication of $\mathrm{CdSe} / \mathrm{CdS}$ heterostructured NCs with unique morphologies [3-22]. CdSe/CdS systems are interesting platforms for elucidating the blinking mechanism because of their "quasi"-type-II band alignments [3-5]. In $\mathrm{CdSe} / \mathrm{CdS}$ core-shell NCs, for example, electrons are delocalized in the $\mathrm{CdS}$ shell, while holes are strongly localized in the CdSe core. An important characteristic resulting from the electron delocalization is a suppression of the Auger recombination process [6-10], which improves the PL quantum efficiencies of excitonic complexes such as charged excitons formed with two electrons and a hole [8,9]. Suppression of the NC charging process is also possible by reducing the probability of the Auger-ionization process in biexcitons, which works as a trigger to initiate blinking $[9,11]$. In addition to these advances, new aspects of $\mathrm{CdSe} / \mathrm{CdS}$ systems are being continuously reported [12-14].

Along with their unique quasi-type-II band alignments, $\mathrm{CdSe} / \mathrm{CdS}$ heterostructured NCs enable us to study interesting structures such as dot-in-rods (a CdSe quantum dot embedded in an elongated $\mathrm{CdS}$ rod) and tetrapods [3,15-22]. In the dot-in-rod structure, electrons are delocalized in the $\mathrm{CdS}$ rod [16-22], which suppresses the Auger process and results in a suppression of blinking [17-19] and the appearance of highly emissive charged excitons [22]. By utilizing unique morphologies of nonspherical dot-in-rod structures, we make it possible to clarify the unlabeled role of electron delocalization on the blinking mechanism. To distinguish the unique characteristics of the dot-in-rod structure from the properties similar to those of core-shell NCs, the introduction of a technique to probe the one-dimensional (1D) nature is

*Corresponding author: kanemitu@scl.kyoto-u.ac.jp
PACS number(s): 78.67.Bf, 42.50.Ar, 78.55.Et, 78.67.Lt necessary. Polarization anisotropy, which can reflect the extent of the electron delocalization [16-19], is a versatile technique. As both PL quantum efficiency and polarization anisotropy are governed by electron delocalization, we expect there to be a certain correlation between the two quantities. Thus, studying the polarization anisotropy of PL from excitonic complexes in dot-in-rods will directly reveal to us the role of electron delocalization during blinking, which would otherwise be difficult to clarify using particles with a spherical morphology.

In this work, we revealed that $\mathrm{CdSe} / \mathrm{CdS}$ dot-in-rods exhibit a unique polarized PL blinking that shows simultaneous changes in the PL intensity, lifetime, and polarization anisotropy under a single-photon emission regime. Variations in the PL polarization anisotropy during blinking are observed, which appear simultaneously with a reduction in the intensity and lifetime, reflecting the charging of the dot-in-rod. We found that there was a significant change in the PL polarization anisotropy for two kinds of charged excitons with different electron delocalizations. We show that the PL polarization degree of excitonic complexes reflects changes of the electron delocalization during blinking, which is governed by the band alignments and the charges both inside and outside the dot-in-rod.

\section{EXPERIMENT}

The CdSe/CdS dot-in-rod samples [Fig. 1(a)] were fabricated according to the synthesis method reported in Ref. [15]. The CdSe core had a diameter of $3.5 \mathrm{~nm}$ and was surrounded by a CdS shell with a thickness of $\sim 5 \mathrm{~nm}$. The CdSe core was located near one end of the CdS rod, which had a radius and length of $6 \mathrm{~nm}$ and 50-60 nm, respectively. Diluted samples were spin coated onto a quartz substrate with or without poly(methyl methacrylate) (PMMA) dissolved in toluene at $0.1 \mathrm{wt} \%$. Monochromatic light pulses at around $510 \mathrm{~nm}$, which were produced by the white-light picosecond pulses from a supercontinuum light source filtered by a monochromator or bandpass filters, were used to excite the samples. The excitation pulse was focused with a spot size of a few micrometers using an objective lens at an excitation power of $\sim 5 \mu \mathrm{W}$. All measurements were conducted at room temperature.

The simultaneous PL intensity, lifetime, and polarization anisotropy measurements were achieved with the optical setup 

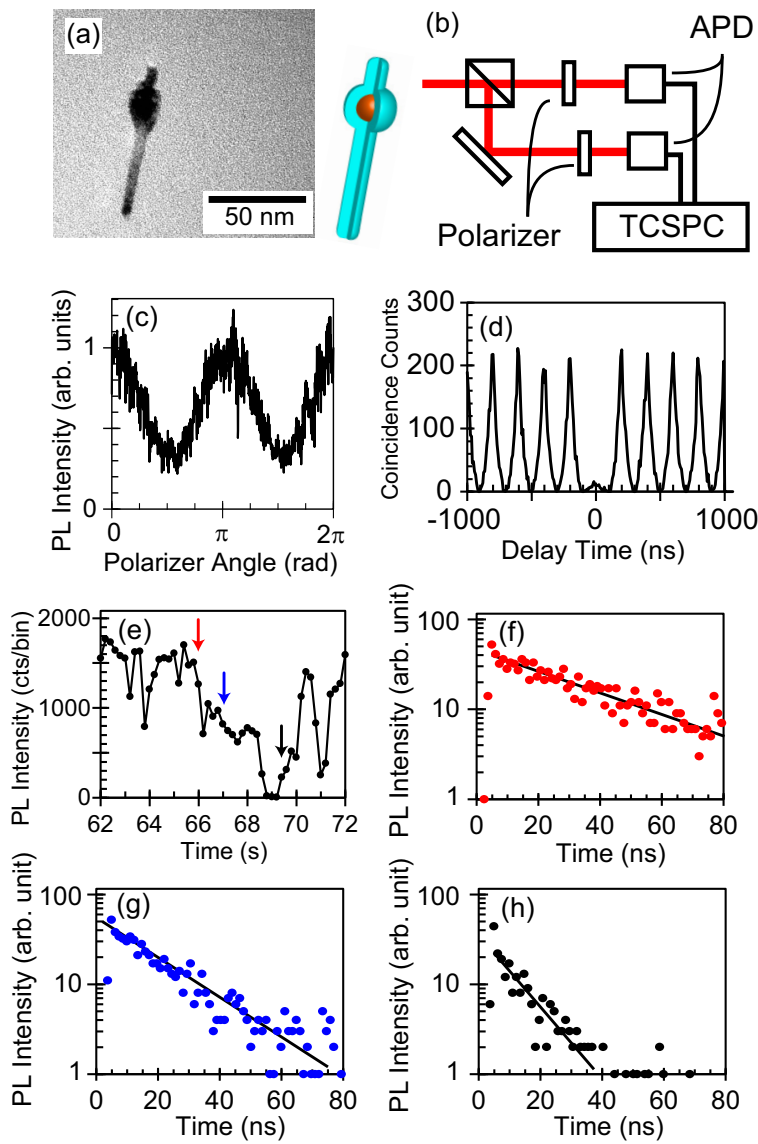

FIG. 1. (Color online) (a) Transmission electron microscope image of a dot-in-rod sample and a schematic of its structure. (b) Detection geometry for measuring the polarization blinking. (c) Polarization-angle dependence of the PL intensity. (d) Coincidence counts as a function of the time delay. (e) An example of the PL intensity time traces showing multiple states. (f)-(h) Decay curves obtained for the data indicated by red, blue, and black arrows in (e).

shown in Fig. 1(b). Two avalanche photodiodes (id100-20 and id100-50; IDQ) were installed in the Hanbury BrownTwiss geometry. Each photon arrival time was recorded using time-correlated single-photon counting boards (SPC-130EM; Becker \& Hickl GmbH) operating under time-tag mode. Two polarizers were placed in front of two detectors to detect the polarized photons.

\section{RESULTS AND DISCUSSION}

Before conducting the polarization blinking measurement, we measured the polarization dependence of the PL intensity so that a sample orientated at an angle almost parallel to the excitation polarization was chosen. The example PL intensity in Fig. 1(c) shows clear cyclic behavior with maxima at $\pi$ and $2 \pi$. After this check of the sample angle, we conducted the photon correlation measurement for $90 \mathrm{~s}$, by setting the angles of both polarizers to record the PL intensity with the polarization parallel to the excitation polarization $\left(I_{\|}\right)$. An example of photon correlation, obtained from the expression of $g^{(2)}(\tau)=\left\langle I_{1}(t) I_{2}(t+\tau)\right\rangle$, is shown in Fig. 1(d). The almost zero coincidence counts at a delay time of $0 \mathrm{~ns}$ confirm
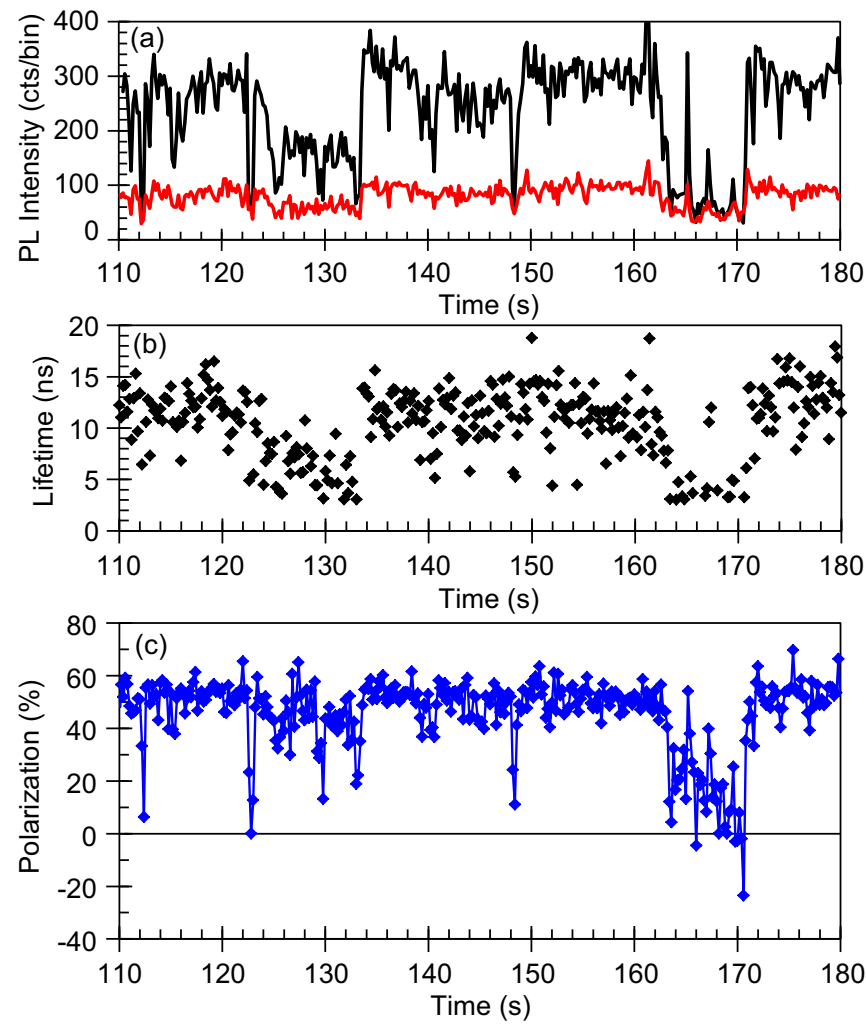

FIG. 2. (Color online) (a) Time traces of the PL intensity obtained with the polarization parallel (black) and perpendicular (red) to the excitation polarization. (b) PL lifetime estimated for each binning time (200 ms) using the parallel polarization data in (a). (c) Time trace of the emission polarization calculated from the data in (a).

the single-photon emission regime and that the sample is truly a single dot-in-rod. After this 90 -s period, one of the polarizers was rotated by $90^{\circ}$ to record the PL intensity with the polarization perpendicular to the excitation polarization $\left(I_{\perp}\right)$.

By using the above procedure, we studied 31 samples that show clear antibunching. All of the samples show at least two states, one state with high emissivity, and one or multiple states with lower emissivity. Figure 1(e) shows an example of PL intensity time trace obtained on one of the detectors for a time range from 62 to $72 \mathrm{~s}$, plotted with a binning time of $200 \mathrm{~ms}$. For signals at 66.0, 67.0, and $68.6 \mathrm{~s}$, where the PL intensities with $1500,1000,300$ counts (cts)/bin were observed, the decay curves are plotted in Figs. 1(f)-1(h), respectively. For all three decay curves, single exponential decays were apparently observed, whose lifetimes became small as the PL intensities decreased. To demonstrate interesting features observed on polarized PL blinking on dot-in-rods, in the following, we show results of two samples exhibiting blinking between three states with different PL polarizations.

Figure 2(a) shows the two PL intensity time traces obtained with one detector recording $I_{\|}$(black) and the other detector recording $I_{\perp}$ (red) for a time range of 110-180 s and a binning time of $200 \mathrm{~ms}$. We see that $I_{\|}$has a large intensity of around $300 \mathrm{cts} / \mathrm{bin}$, for example, at 118-122 s, while $I_{\perp}$ has an intensity of around $100 \mathrm{cts} / \mathrm{bin}$. This difference between $I_{\|}$and $I_{\perp}$ is related to the polarization anisotropy 
observed in Fig. 1(c). The time-dependent changes of $I_{\|}$(e.g., a change from $\sim 300 \mathrm{cts} / \mathrm{bin}$ to $\sim 180 \mathrm{cts} /$ bin at $126-132 \mathrm{~s}$ or to $\sim 50 \mathrm{cts} / \mathrm{bin}$ at $164-170 \mathrm{~s}$ ) correspond to the blinking of the dot-in-rod.

Figure 2(b) shows the corresponding PL lifetime $\left(\tau_{\mathrm{PL}}\right)$ obtained from the $I_{\|}$data. The lifetime was analyed using a single exponential fit in each binning time, which is a common method for studying time-dependent PL lifetimes [8,9,22,23]. For the time periods in which $I_{\|}$is $\sim 300 \mathrm{cts} / \mathrm{bin}$, the $\tau_{\mathrm{PL}}$ is 13 $\pm 2 \mathrm{~ns}$, but it reduces to around $6 \pm 1 \mathrm{~ns}$ and $3 \pm 1 \mathrm{~ns}$ for the periods in which $I_{\|}$is $\sim 180$ and $\sim 50$ cts/bin, respectively. In regards to the high-intensity signal with the longest $\tau_{\mathrm{PL}}$ of $13 \mathrm{~ns}$, we attribute the main contributions to this signal to the photon emission of neutral excitons $(X)$. In contrast, we assign the lower-intensity signals to the photon emission of negatively charged excitons $\left(X^{-}\right)$. Due to an increase of the Auger process by the additional negative charge, the PL quantum efficiencies of the charged excitons are smaller than those of the neutral excitons $[8,9,20,22]$. This model, known as the charging model, explains that the switching between the charged and uncharged NCs is the origin of the abrupt changes of both PL intensities and lifetimes during blinking.

Figure 2(c) shows the polarization anisotropy $(P)$ calculated for each binning time, using the expression $P=$ $\left(I_{\|}-I_{\perp}\right) /\left(I_{\|}+I_{\perp}\right)[16,19]$. For the time periods in which $X$ emission is dominant, the polarization anisotropy is $50 \%$. When $X^{-}$emission is dominant, a $P$ value of $40 \%$ for the periods in which $I_{\|}$is $\sim 180 \mathrm{cts} / \mathrm{bin}$ and a $P$ value of $0 \%-20 \%$ for the periods in which $I_{\perp}$ is $\sim 50$ cts/bin were observed. The charging of the dot-in-rod during blinking represents not only a reduction in the PL intensity and lifetime but also a modification of the emission polarization anisotropy.

To study the correlations between the polarization, intensity, and lifetime, we examined two-dimensional distribution maps of the data. Figure 3(a) shows the sum of the $I_{\|}$and $I_{\perp}$ PL intensities in Fig. 2(a) as a function of the polarization

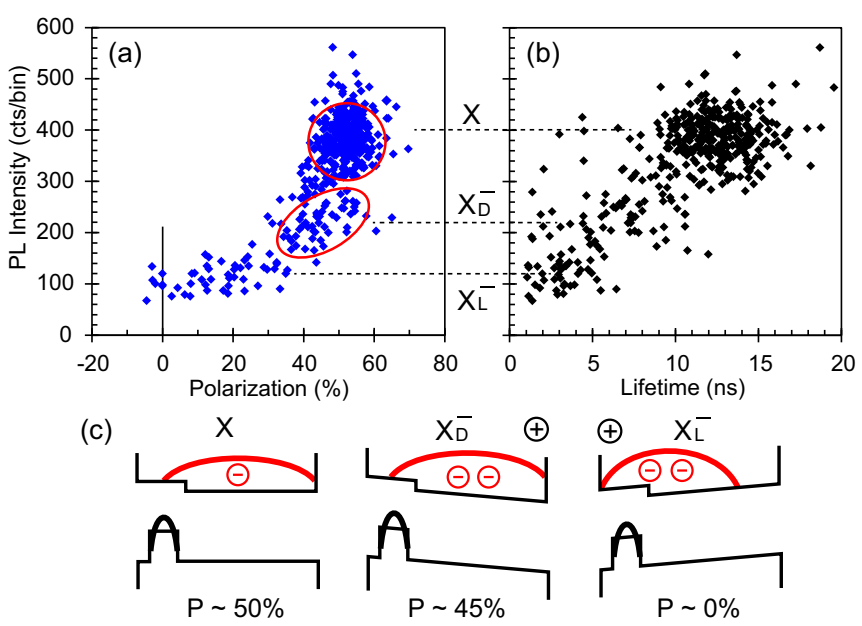

FIG. 3. (Color online) (a) FPID and (b) FLID plots of the data in Figs. 2(a)-2(c). (c) Schematic illustration of the energy diagram of neutral excitons and charged excitons in a dot-in-rod sample with type-II band alignment. The introduction of an electron and the position of the ejected hole change the delocalization of the 1D electron. anisotropy [taken from Fig. 2(c)]; we refer to this as a fluorescence polarization-intensity distribution (FPID) plot. Figure 3(b) shows the PL intensities as a function of the lifetime, i.e., a fluorescence lifetime-intensity distribution (FLID) plot $[8,9,22,23]$. The $X$-emission-dominant signal is indicated by the upper dotted line at a PL intensity of $400 \mathrm{cts} / \mathrm{bin}$ (polarization of $50 \%$ and a lifetime of $13 \pm 2 \mathrm{~ns}$ ), while the two other dotted lines indicate $X^{-}$-emissiondominated signals at PL intensities of 220 and $120 \mathrm{cts} / \mathrm{bin}$. Interestingly, there are clear correlations between the PL intensity, polarization, and lifetime of $X^{-}$; i.e., a high intensity corresponds to a large polarization of $40 \%$ and a long lifetime, while a low intensity corresponds to a small polarization of 0\%-20\% and a short lifetime. The two charged excitons can be distinguished by the significant change in the polarization anisotropy, which occurs simultaneously with a reduction in the intensity and lifetime.

We can interpret these results in terms of the effect of the type-II band alignment, an additional electron in the $\mathrm{NC}$, and an ejected hole. In the neutral exciton state in an uncharged dot-in-rod [Fig. 3(c) left], an electron is strongly delocalized along the $\mathrm{CdS}$ rod resulting in the large $(50 \%)$ polarization anisotropy. In the negatively charged exciton state with an ejected hole at the tip of the CdS rod [Fig. 3(c) center], however, the wave function of the electron is slightly modified by the repulsive Coulomb interaction between two electrons and the attractive potential of the hole outside the NC. Because the electron is delocalized in the $1 \mathrm{D} \mathrm{CdS}$ rod, this state can be denoted as a delocalized charged exciton $\left(X_{D}^{-}\right)$. If the negatively charged exciton state exists in the presence of an ejected hole near the CdSe core [Fig. 3(c) right], then the electron wave function is localized to the CdSe core due to both electron repulsion and hole attraction, and this state can be denoted as a localized charged exciton $\left(X_{L}^{-}\right)$. This model enables us to connect the significant change in the polarization anisotropy to the different $1 \mathrm{D}$ electron delocalizations of $X_{D}^{-}$ and $X_{L}^{-}$; i.e., the large (small) polarization corresponds to delocalized (localized) electrons. Although similar dot-in-rods surrounded by an external charge have been discussed in earlier work [20-22], we present here direct evidence of the excitonic complexes with $1 \mathrm{D}$ electron delocalization during blinking in dot-in-rods. Specifically, while earlier work has reported observations of the surface charge movement outside the dot-in-rods $[20,21]$ and a suppression of the Auger process due to $1 \mathrm{D}$ electron delocalization [22], we have been able to realize a definitive identification of the two charged excitons that have different electron delocalizations. We stress that this was achieved by using our simultaneous measurement technique that allowed us to produce FPID and FLID plots.

The results in Fig. 3 indicate that the sample has a type-II band alignment, with the neutral excitons being strongly delocalized. However, as $\mathrm{CdSe} / \mathrm{CdS}$ heterostructures have quasi-type-II band alignments, their size and shape will determine the band alignment, which can be either type I or type II [3]. In fact, we also obtained experimental results indicating a type-I band alignment, as shown by the FPID and FLID plots in Figs. 4(a) and 4(b), respectively. The main contributions are indicated by the dotted lines at PL intensities of 1200,500 , and $250 \mathrm{cts} / \mathrm{bin}$. We assigned the signal with highest intensity and longest $\tau_{\mathrm{PL}}$ of $10 \pm 2 \mathrm{~ns}$ to $X$, while the 

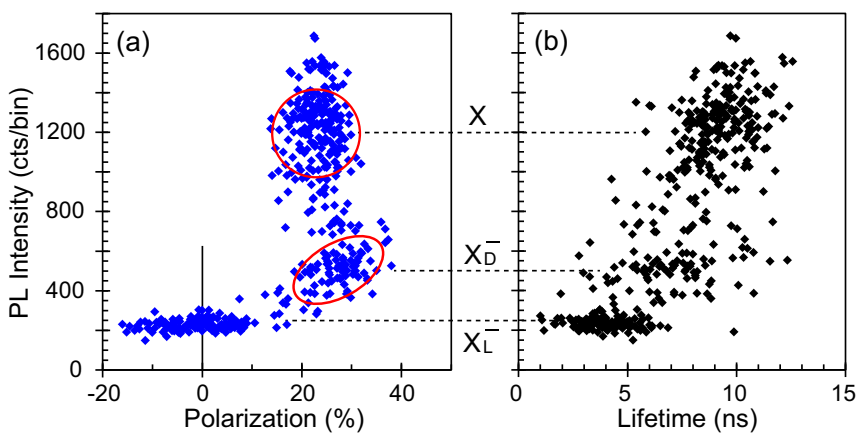

(c)
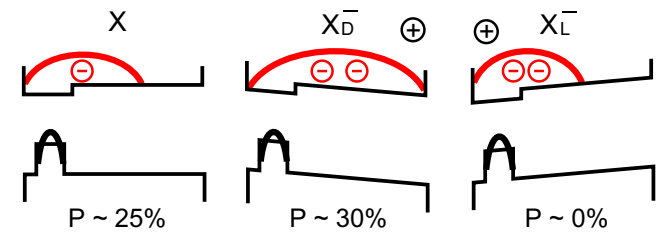

FIG. 4. (Color online) (a) FPID and (b) FLID plots of data from another dot-in-rod sample. (c) Schematic illustration of the energy diagram of neutral excitons and charged excitons in a dot-in-rod sample with type-I band alignment.

other two contributions, with a lower intensity and shorter $\tau_{\mathrm{PL}}$ values of $7 \pm 1 \mathrm{~ns}$ and $4 \pm 1 \mathrm{~ns}$, were assigned to $X_{D}^{-}$and $X_{L}^{-}$, respectively. Interestingly, the polarization of $X_{D}^{-}$was larger than that of $X$, which is in stark contrast to the results shown in Fig. 3(a). This can be explained by considering the type-I band alignment illustrated in Fig. 4(c). When the $\mathrm{NC}$ is uncharged, a neutral exciton is located near the CdSe core with small electron delocalization (left panel), but when the NC is negatively charged and there is an ejected hole at the tip of $\mathrm{CdS}$ rod, the electron is delocalized along the $\mathrm{CdS}$ rod due to both electron repulsion and hole attraction (middle panel). When the ejected hole is closer to the CdSe core, the electron is less delocalized (right panel). This model allows us to connect the change of polarization anisotropy between $X$ and $X_{D}^{-}$to a characteristic of the type-I band alignment. From these considerations of type-II and type-I band alignments in conjunction with charges inside and outside the dot-in-rod, we can conclude that the unique polarized PL blinking of single $\mathrm{CdSe} / \mathrm{CdS}$ dot-in-rods reveals the role of electron delocalization in forming asymmetrically delocalized excitonic complexes in the quasi-type-II band alignment.

It is remarkable that the differences between FPID plots in Figs. 3(a) and 4(a) display a clear contrast between the type-I and the type-II band-alignments; i.e., the polarization of $X_{D}^{-}$signals becomes smaller than that of $X$ signals in Fig. 3(a), while an opposite relation was observed for Fig. 4(a). Among 31 dot-in-rod samples studied in this work, almost half of the samples exhibited change of polarization between $X$ and $X_{D}^{-}$, which include samples indicating typeII band alignments $(\sim 80 \%)$ and samples indicating type-I band alignments $(\sim 20 \%)$. The other half of the samples did not show significant change of polarization between $X$ and $X_{D}^{-}$. When we compare the FLID in Figs. 3(b) and 4(b), we do not observe such a clear contrast between FLID plots. Thus, the FPID plots are a powerful tool for studying electron delocalization. In the case of spherical core-shell NCs with similar quasi-type-II band alignments [4], the different band alignments are hard to probe, as they do not show a large polarization anisotropy. From the same reason, clear identification of the two charged excitons and electron delocalization, i.e., distinguishing between $X_{D}^{-}$and $X_{L}^{-}$, is also difficult to achieve with core-shell NCs. Thus, our approach to studying the blinking of dot-in-rods using an FPID plot has real advantages for understanding the blinking mechanism in various heterostructured NCs. It is also remarkable that definite assignments of $X_{D}^{-}$and $X_{L}^{-}$are important for not only dot-in-rods but also NCs of different morphologies such as core shells and tetrapods. In fact, observations of similar multiple intermediate states, which cannot be explained by single charged excitons, have also been reported for NCs composed of CdSe, CdS, and ZnS [7-9,24-28]. While these intermediate states are often interpreted as doubly charged excitons $[8,9]$ or trapping processes [7-9,24-26], a suitable explanation of the overall blinking mechanism has not yet been provided. We believe that simultaneous measurements of various PL characteristics will be a powerful tool to reveal the unsolved issue of blinking associated with multiple intermediate states in NCs with various morphologies, as we have demonstrated unique properties of charged excitons by FPID and FLID measurements.

\section{CONCLUSION}

In conclusion, we have studied the role of electron delocalization during the blinking of single $\mathrm{CdSe} / \mathrm{CdS}$ dot-in-rods by using simultaneous FPID and FLID measurements. We found that the significant change in the PL polarization anisotropy distinguishes between the two charged excitons with different electron delocalizations. We revealed that the unique polarized PL blinking of single CdSe/CdS dot-in-rods reflects the electron delocalization forming asymmetrically delocalized excitonic complexes in type-I or type-II band alignments.

\section{ACKNOWLEDGMENTS}

Part of this work was supported by KAKENHI (Grant No. 25247052) and JST-CREST. We thank K. Ohno for his help with FLID analyses.
[1] M. Nirmal, B. O. Dabbousi, M. G. Bawendi, J. J. Macklin, J. K. Trautman, T. D. Harris, and L. E. Brus, Nature 383, 802 (1996).

[2] B. Lounis and M. Orrit, Rep. Prog. Phys. 68, 1129 (2005).

[3] N. J. Borys, M. J. Walter, J. Huang, D. V. Talapin, and J. M. Lupton, Science 330, 1371 (2010).
[4] S. Brovelli, R. D. Schaller, S. A. Crooker, F. García-Santamaría, Y. Chen, R. Viswanatha, J. A. Hollingsworth, H. Htoon, and V. I. Klimov, Nat. Commun. 2, 280 (2011).

[5] A. Sitt, F. D. Sala, G. Menagen, and U. Banin, Nano Lett. 9, 3470 (2009). 
[6] P. Spinicelli, S. Buil, X. Quélin, B. Mahler, B. Dubertret, and J.-P. Hermier, Phys. Rev. Lett. 102, 136801 (2009).

[7] D. E. Gómez, J. V. Embden, P. Mulvaney, M. J. Fernée, and H. Rubinsztein-Dunlop, ACS Nano 3, 2281 (2009).

[8] C. Galland, Y. Ghosh, A. Steinbrück, M. Sykora, J. A. Hollingsworth, V. I. Klimov, and H. Htoon, Nature 479, 203 (2011).

[9] C. Galland, Y. Ghosh, A. Steinbrück, J. A. Hollingsworth, H. Htoon, and V. I. Klimov, Nat. Commun. 3, 908 (2012).

[10] M. J. Fernée, C. Sinito, Y. Louyer, C. Potzner, T. Nguyen, P. Mulvaney, P. Tamarat, and B. Lounis, Nat. Commun. 3, 1287 (2012).

[11] Y.-S. Park, A. V. Malko, J. Vela, Y. Chen, Y. Ghosh, F. GarcíaSantamaría, J. A. Hollingsworth, V. I. Klimov, and H. Htoon, Phys. Rev. Lett. 106, 187401 (2011).

[12] C. Javaux, B. Mahler, B. Dubertret, A. Shabaev, A. V. Rodina, Al. L. Efros, D. R. Yakovlev, F. Liu, M. Bayer, G. Camps, L. Biadala, S. Buil, X. Quelin, and J.-P. Hermier, Nat. Nanotechnol. 8, 206 (2013).

[13] O. Chen, J. Zhao, V. P. Chauhan, J. Cui, C. Wong, D. K. Harris, H. Wei, H. Han, D. Fukumura, R. K. Jain, and M. G. Bawendi, Nat. Mater. 12, 445 (2013).

[14] Y. Park, Y. Ghosh, Y. Chen, A. Piryatinski, P. Xu, N. H. Mack, H. L. Wang, V. I. Klimov, J. A. Hollingsworth, and H. Htoon, Phys. Rev. Lett. 110, 117401 (2013).

[15] D. V. Talapin, J. H. Nelson, E. V. Shevchenko, S. Aloni, B. Sadtler, and A. P. Alivisatos, Nano Lett. 7, 2951 (2007).

[16] D. V. Talapin, R. Koeppe, S. Götzinger, A. Kornowski, J. M. Lupton, A. L. Rogach, O. Benson, J. Feldmann, and H. Weller, Nano Lett. 3, 1677 (2003).
[17] F. Pisanello, L. Martiradonna, P. Spinicelli, A. Fiore, J. P. Hermier, L. Manna, R. Cingolani, E. Giacobino, M. De Vittorio, and A. Bramati, Superlattices Microstruct. 47, 165 (2010).

[18] F. Pisanello, L. Martiradonna, G. Leménager, P. Spinicelli, A. Fiore, L. Manna, J. P. Hermier, R. Cingolani, E. Giacobino, M. D. Vittorio, and A. Bramati, Appl. Phys. Lett. 96, 033101 (2010).

[19] A. Sitt, A. Salant, G. Menagen, and U. Banin, Nano Lett. 11, 2054 (2011).

[20] J. Müller, J. M. Lupton, A. L. Rogach, J. Feldmann, D. V. Talapin, and H. Weller, Phys. Rev. Lett. 93, 167402 (2004).

[21] J. Müller, J. M. Lupton, A. L. Rogach, J. Feldmann, D. V. Talapin, and H. Weller, Phys. Rev. B 72, 205339 (2005).

[22] F. T. Rabouw, P. Lunnemann, R. J. A. van Dijk-Moes, M. Frimmer, F. Pietra, A. F. Koenderink, and D. Vanmaekelbergh, Nano Lett. 13, 4884 (2013).

[23] K. Zhang, H. Chang, A. Fu, A. P. Alivisatos, and H. Yang, Nano Lett. 6, 843 (2006)

[24] S. Rosen, O. Schwartz, and D. Oron, Phys. Rev. Lett. 104, 157404 (2010).

[25] J. Zhao, G. Nair, B. R. Fisher, and M. G. Bawendi, Phys. Rev. Lett. 104, 157403 (2010).

[26] P. A. Frantsuzov and R. A. Marcus, Phys. Rev. B 72, 155321 (2005).

[27] N. Amecke and F. Cichos, J. Lumin. 131, 375 (2011).

[28] N. Yoshikawa, H. Hirori, H. Watanabe, T. Aoki, T. Ihara, R. Kusuda, C. Wolpert, T. K. Fujiwara, A. Kusumi, Y. Kanemitsu, and K. Tanaka, Phys. Rev. B 88, 155440 (2013). 